\title{
The Design of Facial expression recognition system based on the LabVIEW and MATLAB mixed programming
}

\author{
Wang $\mathrm{Ju}^{1, a}$, Hong $\mathrm{Mei}^{2, b}$ and Chunyan $\mathrm{Nie}^{3, \mathrm{c}}$ \\ ${ }^{1}$ College of Electronic and Information Engineering, Changchun University, Changchun, 130011, \\ China \\ ${ }^{2}$ College of Electronic and Information Engineering, Changchun University, Changchun, 130011, \\ China \\ ${ }^{3}$ College of Electronic and Information Engineering, Changchun University, Changchun, 130011, \\ China \\ aemail:youyou8221@aliyun.com, bemail: 13849507@qq.com, email: 564934745@qq.com
}

Keywords: LabVIEW; MATLAB; mixed programming; facial expression.

\begin{abstract}
It has a very important role that emotion to coordinate the relationship between people in the numerous and complicated social life. Expression is the most important characteristics of emotion, it is indispensable means of information communication and emotional communication in interpersonal communication. This article is based on the NI company LabVIEW software, using the LabVIEW perfect graphics editing features, structuring the interface of facial expression recognition, and combining with MATLAB powerful engineering computation software, using discrete wavelet to extract the expression images for feature, and last applying of flexible template matching to make the emotion recognition according to the expression.
\end{abstract}

\section{Introduction}

As people understanding of the emotion, emotion research is becoming more and more attention from social, the external behavior of characteristics is emotional expression. If computer can be like humans that have the ability to understand and express emotion that can better serve humanity, which is the facial expression recognition of great significance.

Face recognition technology processes shown in Figure 1. Image acquisition of Japan as the JAFFE image database data, this article focuses on one of the six basic facial expressions, namely: anger, happiness, sadness, surprise, disgust and fear. The first step is face image preprocessing, the next step is to extract from these images expression information that is, expression feature in MATLAB using the corresponding algorithm to extract expression features. Finally, classification and recognition, image output corresponding expression output.

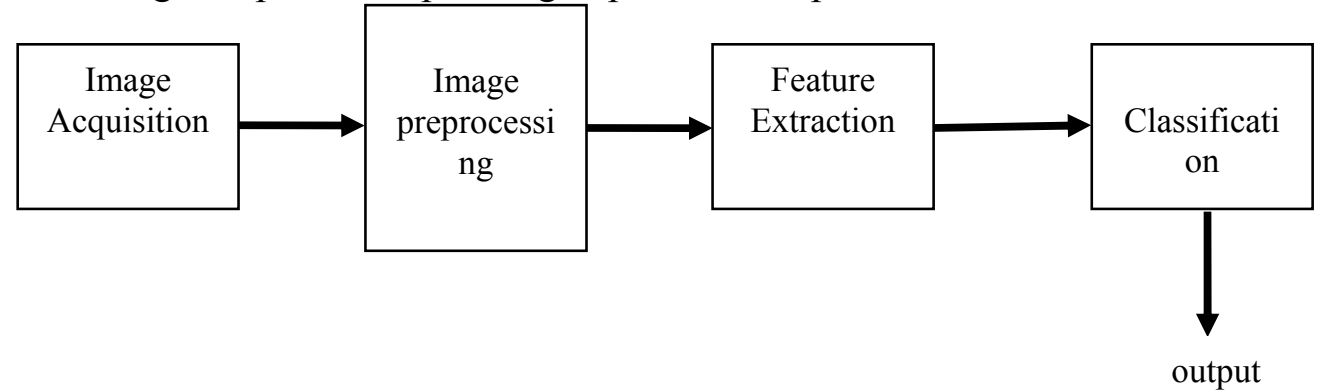

Fig.1. Facial expression recognition chart

\section{Preprocessing of expression}

In order to prevent problems caused by light and other image quality is not high, preprocessing for expression image first, before making facial expression recognition. The recognition system is added 
the expression of image gray-scale extraction respectively, conversion binarized of gray level, and through histogram equalization can adaptive effectively improve the shortage problem of image contrast. We are angry expression, for example, Application of histogram equalization preprocessing under the MATLAB software, unprocessed and processed histogram in Figure2.

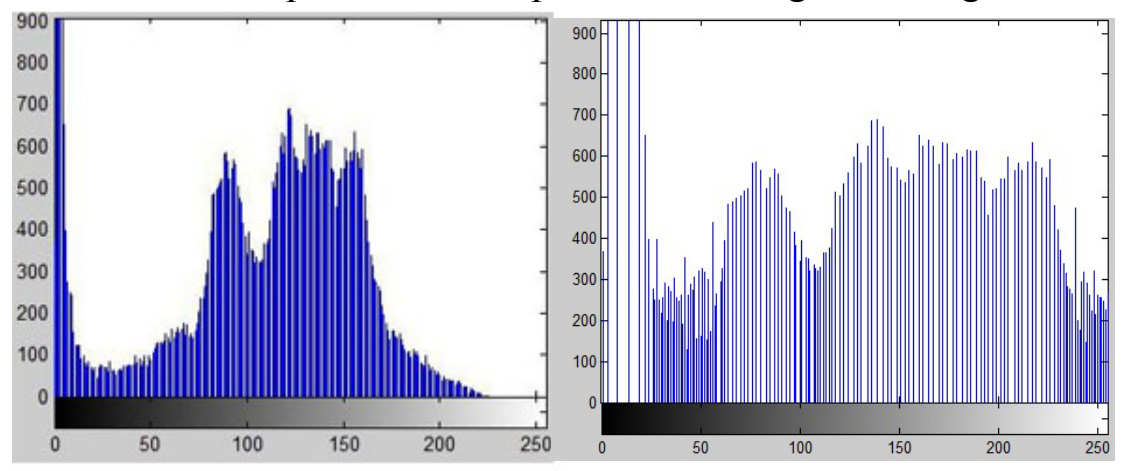

Fig.2. Unprocessed and processed histogram

\section{The feature extraction of image expression}

When multi-resolution digital image observation and processing, discrete wavelet transform is a mathematical tool of choice, in addition to having an effective, highly intuitive description framework and the resolution of the image storage, but also help us understand the spatial domain and frequency image domain characteristics.

When a size of an image of the MN, the discrete transform, can be expressed as:

$T(u, v, \cdots)=\sum_{x, y} f(x, y) g_{u, v, \ldots}(x, y)$

Therefore arbitrary function of discrete wavelet transform is:

$$
f(x, y)=\int_{R} f(t) \overline{\varphi_{m, n}(t)} d_{t}
$$

These six basic expressions for the discrete wavelet transform feature image obtained after the treatment shown in Figure 3. Feature expression processed provides a better feature vector which has the characteristics for classification expression for the latter.

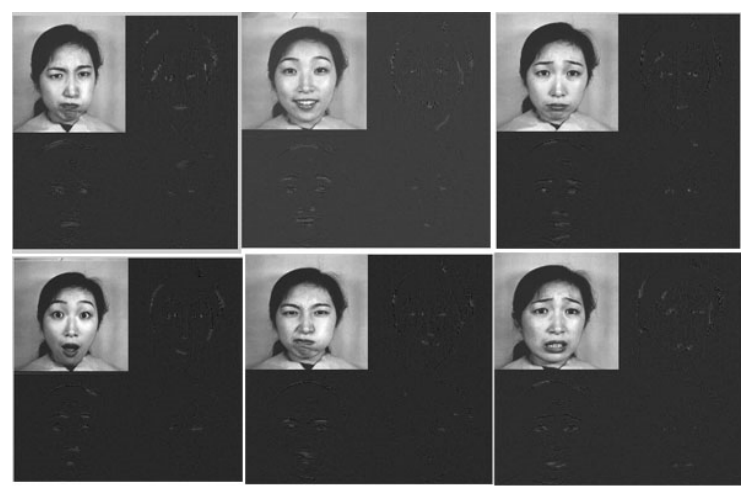

Fig.3. Feature image

\section{Expression Recognition}

The basic idea is: choose some of the image feature points to create a certain elastic template topology, each node extracts a feature vector, defines the similarity function to calculate the topology of the feature vector and the similarity, the similarity by pursuing maximization to complete. Elastography feature point shown in Figure 4. 


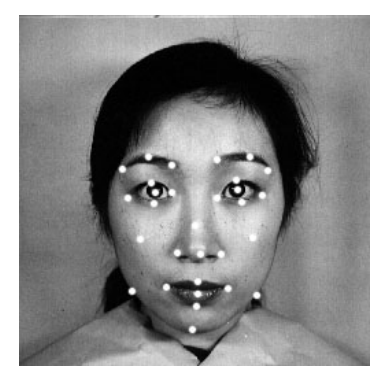

Fig.4. Elastography feature points

\section{The realization of facial expression recognition system}

This paper is the application of MATLAB and LabVIEW software mixed programming, Embedding MATLAB for expression processing into LabVIEW. The MATLAB and LabVIEW software are two of the most popular software in engineering computation currently. LabVIEW is the NI company development program based on graphical programming language, it's clear and concise graphical programming language. Through LabVIEW software design, making the facial expression recognition system interface become more colorful. In this paper, there are three options panel application of the LabVIEW software design, it can perform three steps: image acquisition, image preprocessing and expression to identify. The front panel of image acquisition is shown in figure 5 . The front panel of image preprocessing as shown in figure 6, the front panel facial expression recognition as shown in figure7.

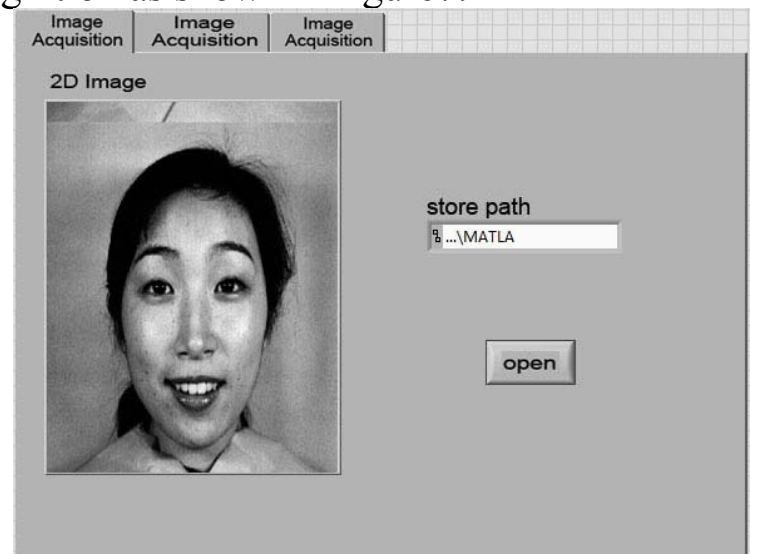

Fig.5. The front panel of image acquisition preprocessing

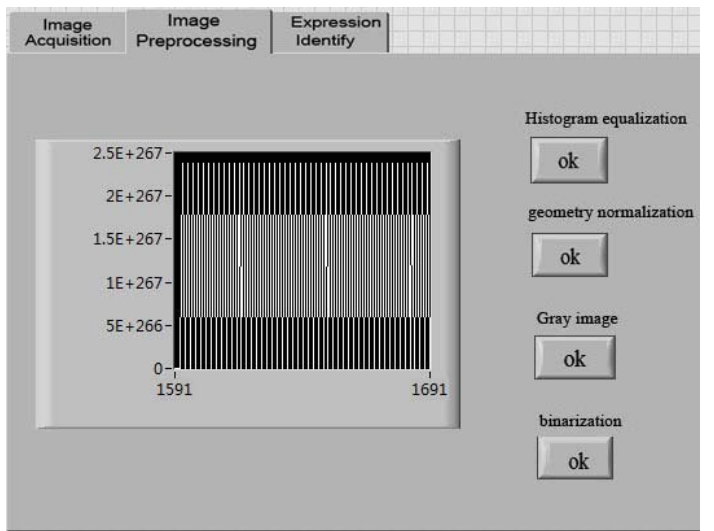

Fig.6. The front panel of image

\section{LabVIEW call MATLAB program}

MATLAB is a proficient in engineering and scientific computing software, this paper makes use of its powerful function of image processing, to preprocess acquired the expression of image. In this paper, the key step is to call MATLAB procedures in LabVIEW, apply the wavelet analysis toolbox of discrete wavelet analysis within it to get the expression image features. Call the process of the function in LabVIEW (function) module - mathematics - formula - MATLAB script node, the part program which LabVIEW calling MATLAB program as shown in figure 8. 

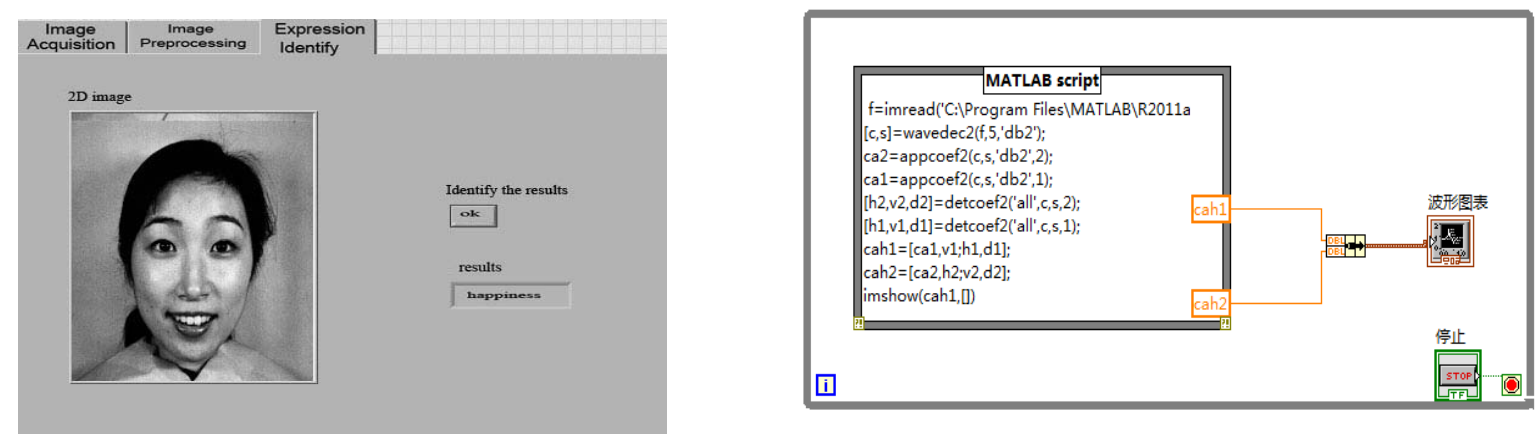

Fig.7. the front panel facial expression recognition Fig.8. LabVIEW call MATLAB program

\section{Summary}

In this paper, the facial expression recognition system we study is built on the basis of the two engineering software LabVIEW and MATLAB mixed applications. Application of LabVIEW rich perfect graphical software to create system interface, application of wavelet analysis toolbox of MATLAB software to preprocessing expression image and feature extraction. The LabVIEW vivid graphic language and MATLAB powerful graphics functions perfect unifies in together. Finally apply elastic template matching method identify the corresponding expressions, the recognition rate is above $85 \%$.

\section{Acknowledgement}

In this paper, the research was sponsored by the Department of Education of Jilin Province (Project 2013No. 458).

\section{References}

[1]BruceV. What the human face tells the human mind: some challenges for the robot-human interface. Proceedings. IEEE International Workshop on Robot and Human Conununication.ToKyo,1992:44-45

[2\} Takeuchi A, Nagao K. Communicative facial displays as a new conversational modality Proceedings of INTERCHI'93 Conference on Human Factors in Computing Systems Amsterdam, 1993:187-193

[3] Hara F, Kobayashi H, state of the art in component development for interactive Communication with humans. Advanced Robotics, 1997, 11(6):585-604

[4]Nagamachi M. Kansei Engineering: A new ergonomic consumer-oriented technology for Product development[J]. International Journal of Industrial Ergonomics, 1995.

[5]B.Kort, R.Reilly, R.Pcard. An affective model of interplay between emotions and Learning: reengineering educational Pedagogy-building a Learning companion[C].IEEE International Conference on Advanced Learning Technologies.2001.

[6]Pinhanez C S. The everywhere displays projector: a device to create ubiquitousGraphical interfaces [C]. Proceedings of the $3^{\text {rd }}$ International Conference on Ubiquitous Computing, 2001, 315-331.

[7]Picard R W, Computers that Recognise and Respond to User Emotion: Theoretical and practical Implications, MIT Media Lab Tech Report, 1995. 